\title{
Nonlinear optical fiber polarization tracking at 200 $\mathrm{krad} / \mathrm{s}$
}

\author{
Victor V. Kozlov ${ }^{1,2}$, Julien Fatome ${ }^{3}$, Philippe Morin ${ }^{3}$, Stephane Pitois ${ }^{3}$, and Stefano \\ Wabnitz ${ }^{1}$ \\ ${ }^{1}$ Department of Information Engineering, Università di Brescia, \\ Via Branze 38, 25123 Brescia, Italy \\ ${ }^{2}$ Department of Physics, St.-Petersburg State University, \\ Petrodvoretz, St.-Petersburg, 198504, Russia \\ ${ }^{3}$ Laboratoire Interdisciplinaire Carnot de Bourgogne, UMR 5209 CNRS / Université de Bourgogne 9 av. Alain \\ Savary, 21078 Dijon, France \\ victor.kozlov@email.com
}

\begin{abstract}
We demonstrate endless nonlinear polarization stabilization with a standard telecom fiber at a tracking speed of $200 \mathrm{krads} / \mathrm{s}$. We provide a simple analytical estimate for the response time of nonlinear polarization control.
\end{abstract}

OCIS codes: $230.1150,230.5440$

\section{Introduction}

The state of polarization (SOP) at the output of optical fiber links randomly fluctuates in time due to environmental variations (e.g., temperature) or mechanical shocks which may induce rotation speeds on the Poincare sphere of up to $500 \mathrm{krads} / \mathrm{s}$ [1]. In the presence of polarization-sensitive components at the end of the link such as coherent receivers, optical switches, polarization demultiplexers or sensors, the stabilization of the SOP at the fiber output requires endless or continuous polarization control devices with relatively high tracking speed. With conventional polarization controllers based on Lithium-Niobate electro-optical polarization transformers, polarization tracking speeds have risen from $0.1 \mathrm{rads} / \mathrm{s}$ [2] up to $59 \mathrm{krads} / \mathrm{s}$ [3]. A new class of all-fiber polarization controllers has been proposed in recent years, based on nonlinear polarization interactions, including so-called Brillouin and Raman polarizers, Refs. [4,5], which may enable ultrafast polarization tracking and control. Since the response time of fiber nonlinearity is virtually instantaneous, all-optical polarization control over the bit (or symbol) period (i.e., on a sub-ns time scale) may be envisaged, thus permitting the full compensation of major current transmission impairments such as cross-phase modulation induced polarization scattering. Among different nonlinear polarization control devices, those based on cross-polarization modulation [6,7] in optical fibers are most relevant to us here. This type of devices (also known as nonlinear lossless polarizer, or NLP) is particularly interesting in that they permit to transform any input SOP into virtually one and the same SOP at the fiber output without any polarization-dependent losses. Moreover, the NLP is a broadband device since it may permit the simultaneous control of the SOP of a comb of WDM channels. A practical NLP is implemented by means of a span of a low-PMD optical fiber which is counter-pumped by a fully polarized $\mathrm{CW}$ beam [7]. The output signal SOP is fixed by the SOP of the pump beam.

It is important to note that in a NLP the signal beam must be weaker but still of comparable intensity to the CW pump. Therefore in order to obtain significant repolarization the length of the fiber span should accommodate a few nonlinear lengths, where the nonlinear length $L_{N L}=\left(\gamma P_{S}\right)^{-1}$ ( $\gamma$ is the Kerr coefficient) is defined with respect to the power of the signal beam $P_{S}$. Motivated by the observation in ref. [7] that a NLP can effectively annihilate individual microsecond long input polarization bursts, in this work we unveil what is the ultimate tracking speed of these devices which results in endless compensation of short input SOP polarization fluctuations. In other words, we provide an answer to the question: how fast the SOP fluctuations can be in order to maintain the high performance of a NLP? We argue that the characteristic NLP response time is simply $T_{N L}=L_{N L} / v$ ( $v$ is group speed of light in the fiber). Fluctuations that are slower than $T_{N L}$ are repolarized by the NLP, while the NLP cannot perform properly when the fluctuations become comparable or faster than $T_{N L}$. Our experiments demonstrate a polarization tracking speed of up to $200 \mathrm{krads} / \mathrm{s}$, in good agreement with numerical simulations. 


\section{Results}

Figure 1 illustrates the experimental setup. The polarization attraction process takes place in a 6.2-km long Non-Zero Dispersion-Shifted Fiber (NZDSF). Its parameters are: chromatic dispersion $D=-1.5 \mathrm{ps} / \mathrm{nm} / \mathrm{km}$ at $1550 \mathrm{~nm}$; Kerr coefficient $1.7 \mathrm{~W}^{-1} \mathrm{~km}^{-1}$; polarization mode dispersion (PMD) $0.05 \mathrm{ps} / \mathrm{km}^{1 / 2}$. The $0.7 \mathrm{~W}$ signal $(1.1 \mathrm{~W}$ counterpropagating pump) wave consists of a polarized incoherent wave with spectral linewidth of $100 \mathrm{GHz}$ and a central wavelength of $1544 \mathrm{~nm}(1548 \mathrm{~nm})$. The spectral linewidth of the signal and pump waves is large enough to avoid any Brillouin backscattering. The pump wave has a fixed SOP. A polarization scrambler introduces random polarization fluctuations in the input signal. The signal is amplified by means of an EDFA before its injection into the NZDSF. At the fiber output, the signal SOP is analyzed in terms of its Stokes vectors by means of a polarization analyzer.

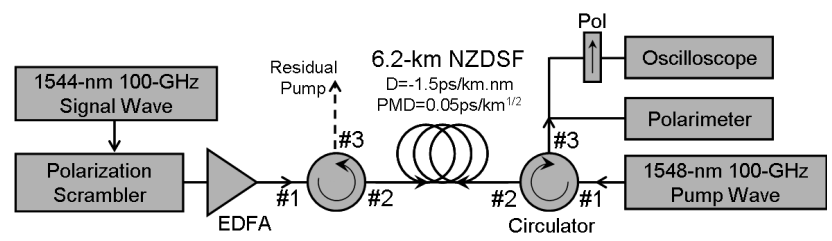

Fig. 1. Experimental setup.

Fig. 2 illustrates the polarization attraction efficiency. At the input end of the fiber, the SOP of the signal randomly fluctuates on a ms-scale owing to the polarization scrambler. This leads to a set of $N=128$ fully polarized beams with a random SOP distribution: all input Stokes vectors are uniformly distributed on the Poincaré sphere (Fig. 2a). Whenever the counter-propagating pump wave is injected (Fig. 2b) we observed an efficient polarization attraction process in the NZDSF. Indeed the small area of the output polarization fluctuations shows that the SOP of the signal was efficiently stabilized. The degree of polarization (DOP) of the signal was thus increased by the NLP from 0.15 in Fig. 2a to 0.99 in Fig. 2 b.

(a)

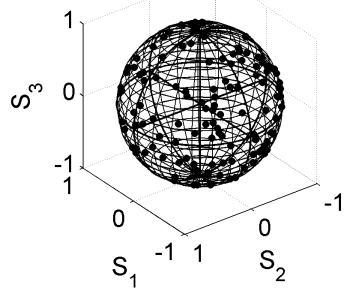

(b)

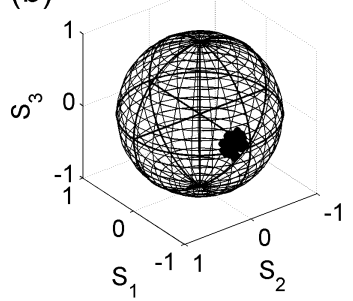

Fig. 2. a) SOP of the input signal (b) SOP of the output signal.

In order to experimentally investigate the transient stage of the attraction process, we recorded the evolution of a fast polarization event as a function of signal power, that is to say as a function of $T_{N L}$. More precisely, a $30 \mu$ s polarization burst was generated on the signal wave by means of the polarization scrambler. The burst was then injected into the fiber along with the counter-propagating pump wave. At the fiber output we detected the burst profile in the time domain thanks to a polarizer. The evolution of the burst was recorded on orthogonal polarization directions as a function of signal power for a constant pump power of $1.1 \mathrm{~W}$. The experimental results are illustrated in Fig. 3(a,b). Note the perfect complementarity between the evolution of the burst on both axes, which provide a general overview of the attraction process. At low signal powers $(10 \mathrm{~mW})$, the nonlinear length $(55 \mathrm{~km})$ is much longer than the fiber length $(6 \mathrm{~km})$ and no attraction process develops. As the signal power grows larger, the nonlinear length decreases until it reaches the fiber length for signal powers around $70 \mathrm{~mW}$. At this point the nonlinear response time $T_{N L}$ is close to the burst duration and the attraction process begins to develop. A transient regime can then be observed with the formation of a short spike on the falling edge of the burst and even slight oscillations, in good agreement with the numerical predictions of Fig. 3(c). As the signal power is further increased, the nonlinear length decreases below $1 \mathrm{~km}$ and the polarization attraction process acts in full strength in order to entirely annihilate the polarization burst. 
(a) $0^{\circ}$

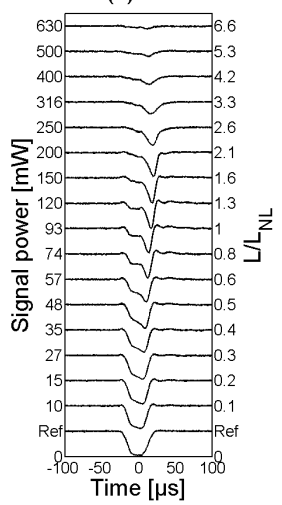

(b) $90^{\circ}$

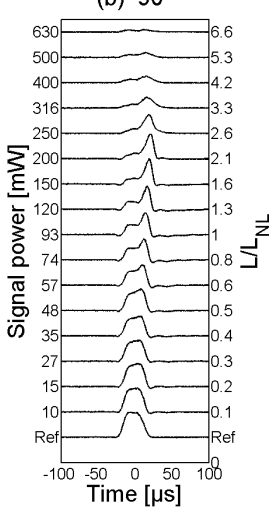

C)

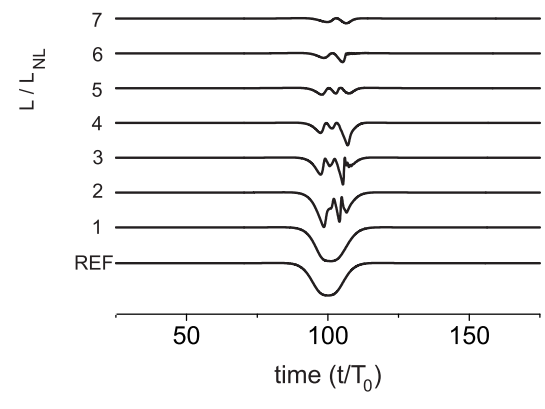

Fig. 3. Evolution of a signal polarization burst as a function of signal average power as detected at the fiber output behind a polarizer along the (a) $0^{\circ}$ axis (b) orthogonal axis. c) Numerical simulation of the $0^{\circ}$ axis case. $T_{0}=L / v(L-$ total fiber length $)$.

The numerical simulations of Fig. 3(c) were performed by solving the coupled Stokes vector equations for the signal and pump [8]: $\partial_{\xi} \mathbf{S}^{s}=\mathbf{S}^{s} \times J_{x} \mathbf{S}^{p}$ and $\partial_{\eta} \mathbf{S}^{p}=\mathbf{S}^{p} \times J_{x} \mathbf{S}^{s}$, where $\xi=(v t+z) / 2$ and $\eta=(v t-z) / 2$. The sign " $\times$ " denotes vector product. The cross-polarization modulation tensor $J_{x}=\frac{8}{9} \gamma \operatorname{diag}(-1,1,-1)$.

\section{Conclusion}

The response time of a NLP based on counter-propagating beams in a low-PMD transmission fiber can be characterized in terms of the $T_{N L}$ parameter. We demonstrated polarization tracking speeds of $200 \mathrm{krads} / \mathrm{s}$, that is about four times the current record for electrooptical polarization control devices. For signal powers around $1 \mathrm{~W}$ and typical nonlinear coefficients $\gamma \sim 1(\mathrm{~W} \cdot \mathrm{km})^{-1}$, the nonlinear length $L_{N L} \sim 1 \mathrm{~km}$ and the corresponding response time $T_{N L} \sim 3 \mu \mathrm{s}$, so that endless polarization control at Mrads/s speeds is feasible using standard telecom fibers. This speed could potentially be increased into the Grads/s regime by using relatively short lengths of special (i.e., highly nonlinear or birefringent) fibers (see Refs. [6]).

The work of V.V. Kozlov and S. Wabnitz was sponsored by MIUR (Ministero dell' Istruzione, dell'Università e della Ricerca) in the framework of the project "Nonlinear cross-polarization interactions in photonic devices and systems".

\section{References}

1. M. Reimer, D. Dumas, G. Soliman, D. Yevick, and M. OSullivan, Polarization evolution in dispersion compensation modules, presented at the Optical Fiber Communications / National Fiber Optic Engineers Conference (OFC/NFOEC), San Diego, CA, March 2226, 2009, Paper OWD4

2. R. Noe, H. Heidrich, and D. Hoffmann, "Endless polarization control systems for coherent optics", J. Lightwave Technol. 6, 1199-1207 (1988)

3. B. Koch, R. Noe, V. Mirvoda, H. Griesser, S. Bayer, H. Wernz, ”Record 59-krad/s Polarization Tracking in 112-Gb/s 640-km PDM-RZ-DQPSK Transmission", IEEE Photonics Technol. Lett. 22, 1407-1409 (2010)

4. A. Zadok, E. Zilka, A. Eyal, L. Thévenaz, and M. Tur, "Vector analysis of stimulated Brillouin scattering amplification in standard single-mode fibers," Opt. Express 16, 21692-21707 (2008)

5. M. Martinelli, M. Cirigliano, M. Ferrario, L. Marazzi, P. Martelli, Evidence of Raman-induced polarization pulling, Opt. Expr. 17, 947-955, 2009.

6. S. Pitois, J. Fatome, and G. Millot, "Polarization attraction using counter-propagating waves in optical fiber at telecommunication wavelengths," Opt. Express 16, 6646-6651 (2008).

7. J. Fatome, S. Pitois, P. Morin, and G. Millot, "Observation of light-by-light polarization control and stabilization in optical fibre for telecommunication applications," Opt. Express 18, 15311-15317 (2010).

8. V. V. Kozlov, J. Nun̄o, and S. Wabnitz, "Theory of lossless polarization attraction in telecommunication fibers," J. Opt. Soc. Am. B 28, 100-108 (2011). 